\title{
Experimental tests of QCD scaling laws at large momentum transfer in exclusive light-meson photoproduction
}

\author{
Moskov J. Amaryan $\odot,{ }^{1}$ William J. Briscoe $\odot,{ }^{2}$ Michael G. Ryskin $\odot,{ }^{3}$ and Igor I. Strakovsky $\oplus^{2, *}$ \\ ${ }^{1}$ Department of Physics, Old Dominion University, Norfolk, Virginia 23529, USA \\ ${ }^{2}$ Institute for Nuclear Studies, Department of Physics, The George Washington University, Washington, DC 20052, USA \\ ${ }^{3}$ Petersburg Nuclear Physics Institute, NRC Kurchatov Institute, Gatchina, St. Petersburg 188300, Russia
}

(Received 10 February 2021; revised 15 April 2021; accepted 10 May 2021; published 21 May 2021)

\begin{abstract}
We evaluated CLAS Collaboration measurements for the $90^{\circ}$ meson photoproduction off the nucleon using a tagged photon beam spanning the energy interval $s=3-11 \mathrm{GeV}^{2}$. The results are compared with the "quark counting rules" predictions.
\end{abstract}

DOI: 10.1103/PhysRevC.103.055203

\section{INTRODUCTION}

Binary reactions in QCD with large momentum transfer involve quark and gluon exchanges between colliding particles. The quark counting rule (QCR) of Brodsky and Farrar [1] and Matveev, Muradyan, and Tavkhelidze [2] has a simple recipe to predict the energy dependence of the differential cross sections of two-body reactions at large meson production angles when $t / s$ is finite and is kept constant. The fixed production or scattering angle behavior for exclusive processes is expected to be $[3,4]$

$$
d \sigma / d t(s) \propto s^{-(n-2)},
$$

where $n$ is the minimum number of fundamental constituents (quarks) and $s, t$, and $u$ are Mandelstam variables.

Recall that in order to provide the exclusivity of hard scattering, we have to balance the large transferred momentum between all the quarks in the hadron. This means that, in order to get the maximum contribution, we have to consider the Fock components of the hadron wave function with the minimum number of quarks. Moreover, these quarks should be close to each other. Small quark-quark separation provides the possibility to better balance the momenta between the quarks. These two conditions are the basis of the QCR expression (1). In the case of Ref. [2], the authors consider just the probability of finding the quarks sufficiently close to each other while in Ref. [1] the balance of the quark momenta was reached via the exchange of an additional gluon between the quarks. Since the virtuality of this gluon is large it means that again we consider the configuration with a short range quark-quark configuration.

\footnotetext{
${ }^{*}$ Corresponding author: igor@gwu.edu
}

Published by the American Physical Society under the terms of the Creative Commons Attribution 4.0 International license. Further distribution of this work must maintain attribution to the author(s) and the published article's title, journal citation, and DOI. Funded by $S C O A P^{3}$.
After the work of Brodsky and Lepage [3], the hard elastic scattering in QCD and the corrections to the leading behavior were intensively discussed (see, e.g., [5-7] and the references therein). If the photon is assumed to be one elementary field, then the prediction for meson photoproduction is

$$
d \sigma / d t(s) \propto s^{-7} .
$$

For the hadron-proton interaction, the counting rule works well, where the hadron is a pion, kaon, proton, or antiproton [4,8-11]. The light-meson photoproduction was examined in terms of the counting rule in Refs. [12-22]. As first observed at SLAC by Anderson et al., the reaction $\gamma p \rightarrow \pi^{+} n(s=$ 8.4-15 $\mathrm{GeV}^{2}$ ) shows agreement with constituent counting rules that predict the cross section should vary as $s^{-7}$ and $(n-2)=7.3 \pm 0.4$ [12]. The agreement extends down to $s=$ $6 \mathrm{GeV}^{2}$, where baryon resonances are still playing a role. ${ }^{1}$.

Note, however, that the quark counting rules account for the minimum numbers of elementary hard processes needed to provide a large momentum transfer to the hadron. At a very large energies, these rules are modified by the so-called Sudakov form factor [24].

Indeed, it is very improbable that two ensembles of constituents can get a strong transverse kick and radiate no gluons. Of course, the probability of a new gluon emission is suppressed by the QCD coupling constant $\alpha_{s}$, but simultaneously it can be enhanced by the square of the large logarithm, $\ln ^{2} s$. The probability of not emitting any additional gluons is called the Sudakov form factor. Thus for a very large $s$, we expect that the cross section of the large angle hadron-hadron scattering should fall with $s$ faster than the QCR prediction [5,6]. However, in an exclusive hard-scattering amplitude the hadron participates as a small color-singlet configuration where its constituent quarks are at short distances. This property of hadron physics suppresses gluon radiation and effects

${ }^{1}$ Photoproduction of $K$ mesons was considered in terms of QCR in Ref. [23] 


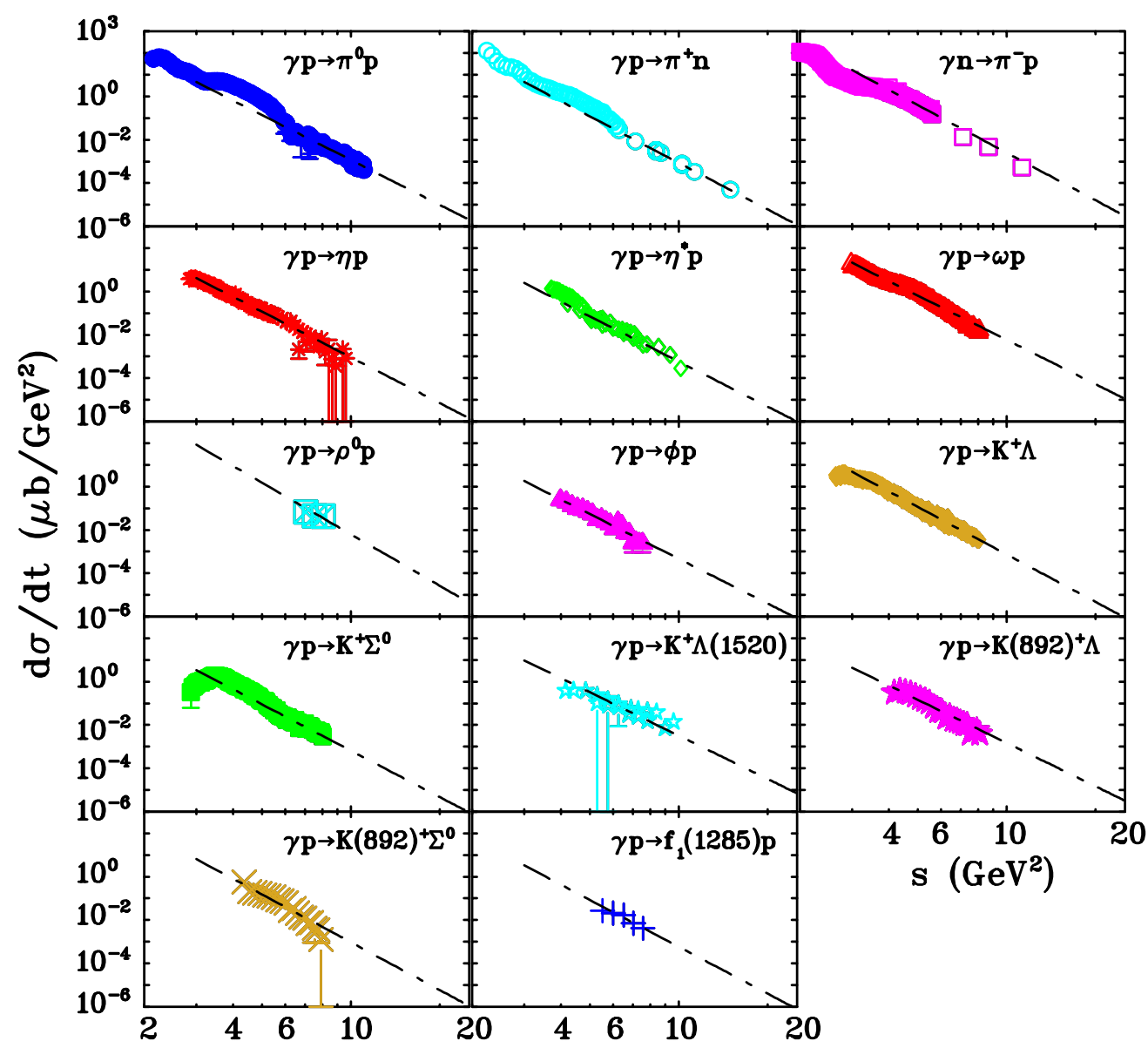

FIG. 1. Differential cross section of $\gamma N \rightarrow M B, d \sigma / d t$, at large meson production angle $\theta=90^{\circ}$ in c.m. as a function of invariant energy squared, $s$ (here $M$ is a meson and $B$ is a baryon). Data are $\gamma p \rightarrow \pi^{0} p[21,28]$ (blue filled circles), $\gamma p \rightarrow \pi^{+} n$ [12,15,29] (cyan open circles), $\gamma p \rightarrow \pi^{-} p$ [15,30] (magenta open squares), $\gamma p \rightarrow \eta p$ [31,32] (red open asterisks), $\gamma p \rightarrow \eta^{\prime} p$ [32-34] (green open diamonds), $\gamma p \rightarrow \omega p$ [16,35] (red open triangles), $\gamma p \rightarrow \rho^{0} p$ [14], (cyan open squares with crosses), $\gamma p \rightarrow \phi p$ [36] (magenta filed triangles), $\gamma p \rightarrow K^{+} \Lambda$ [37] (yellow filled diamonds), $\gamma p \rightarrow K^{+} \Sigma^{0}$ [38] (green filled squares), $\gamma p \rightarrow K^{+} \Lambda(1520)$ [39,40] (cyan open stars), $\gamma p \rightarrow K(892)^{+} \Lambda$ [41] (magenta filled stars), $\gamma p \rightarrow K(892)^{+} \Sigma^{0}$ [41] (yellow crosses), and $\gamma p \rightarrow f_{1}$ (1285) $p$ [33] (blue crosses). The black dash-dotted line is a result of the best fits summarized in Table $\mathrm{I}$. In the case of the $\omega$, the result corresponds to the full energy range, $s=3.5-8.1 \mathrm{GeV}^{2}$.

of the Sudakov form factor. Still the role of Sudakov form factor in large angle $p p$ elastic scattering is not negligible. It was considered in Refs. [25,26].

On the other hand, it was shown in Ref. [27] that, due to the pointlike- nature of the photon, the Sudakov form factor is absent in the case of large angle photoproduction. Thus, photoproduction allows one to check the QCR directly in its original form.

In the present paper, we examined how the counting rules are applicable to the lightest meson photoproduction off the nucleon up to $s=11 \mathrm{GeV}^{2}$, where modern data are available, mostly produced by the CLAS Collaboration at Jefferson Laboratory. The minimum value of $s$ in all these data exceeds 3 $\mathrm{GeV}^{2}$.

Recall that there are three options of how one can consider a photon when it interacts with a nucleon:

(1) No constituents $\left(n_{\gamma}=0\right)$ or $d \sigma / d t(s) \propto s^{-6}$.

(2) The photon is a pointlike particle which participates in the strong interaction $\left(n_{\gamma}=1\right)$ or $d \sigma / d t(s) \propto s^{-7}$.
(3) There is a $q-\bar{q}$ configuration which actually participates in the interaction $\left(n_{\gamma}=2\right)$ or $d \sigma / d t(s) \propto s^{-8}$

\section{LIGHT-MESON PHOTOPRODUCTION REACTIONS}

The JLab6 era has ended at Jefferson Laboratory, leaving in its wake a plethora of cross section measurements for lightmeson photoproduction off the nucleon. There is a unique opportunity to bridge the resonance and high-energy regions, in particular encompassing the region in which "Regge" theory is applicable, and to evaluate the quark counting rule phenomenology with differential cross sections above the "resonance" energies.

The new CLAS high statistical cross sections, for instance, obtained recently for $\gamma p \rightarrow \pi^{0} p$ [21] are compared in Fig. 1 (top left) with previous data from CLAS measurements [28]. At higher energies (above $s \approx 6 \mathrm{GeV}^{2}$ ) and large pion production angles $\left(\theta=90^{\circ}\right)$ in the center-of-mass (c.m.) frame, the results are consistent with the $s^{-7}$ scaling expected from the QCR. The black dash-dotted line is a result of the best-fit of 
TABLE I. Power factor $(n-2)$ in Eq. (1) for light-meson photoproduction off the nucleon from the CLAS Collaboration. The top (bottom) part summarizes pseudoscalar-meson (vector-meson) results. The first column gives reactions and the fourth column shows best-fit results for the energy $s$ ranges listed in the second column and $|t|$ ranges given in the third column. Sources are given in the fifth column. To perform the best fit for $\gamma p \rightarrow \pi^{+} n$, we added SLAC data [12] to JLab Hall A measurements [15,29]. As seen from Fig. 1, in the case of vector-meson production at a larger $s$ the power $(n-2)$ increases. This can be explained by the fact that, in order to form the vector meson, we need to flip the $s$-channel helicity of the quark, as mentioned in the text below.

\begin{tabular}{lcccc}
\hline \hline Reaction & $\begin{array}{c}s \\
\left(\mathrm{GeV}^{2}\right)\end{array}$ & $\begin{array}{c}|t| \\
\left(\mathrm{GeV}^{2}\right)\end{array}$ & $(n-2)$ & Ref. \\
\hline$\gamma p \rightarrow \pi^{0} p$ & $5.9-11.1$ & $2.1-4.7$ & $6.89 \pm 0.26$ & {$[21]$} \\
$\gamma p \rightarrow \pi^{+} n$ & $6.3-14.9$ & $2.3-6.6$ & $7.14 \pm 0.22$ & {$[12,15,29]$} \\
$\gamma n \rightarrow \pi^{-} p$ & $4.0-11.3$ & $0.2-4.6$ & $7.29 \pm 0.14$ & {$[15,30]$} \\
$\gamma p \rightarrow \eta p$ & $3.2-9.6$ & $0.6-3.8$ & $7.02 \pm 0.16$ & {$[31]$} \\
$\gamma p \rightarrow \eta^{\prime} p$ & $4.2-9.3$ & $0.8-2.6$ & $6.92 \pm 0.22$ & {$[32-34]$} \\
$\gamma p \rightarrow K^{+} \Lambda$ & $4.0-8.0$ & $0.3-2.9$ & $7.28 \pm 0.06$ & {$[37]$} \\
$\gamma p \rightarrow K^{+} \Sigma^{0}$ & $5.2-8.0$ & $0.3-2.8$ & $7.12 \pm 0.21$ & {$[38]$} \\
$\gamma p \rightarrow K^{+} \Lambda(1520)$ & $4.8-7.8$ & $0.9-3.2$ & $6.65 \pm 0.41$ & {$[39,40]$} \\
$\gamma p \rightarrow \omega p$ & $3.5-8.1$ & $0.3-2.9$ & $6.80 \pm 0.11$ & {$[16,35]$} \\
$\gamma p \rightarrow \omega p$ & $5.0-8.1$ & $0.3-2.9$ & $8.80 \pm 0.06^{\mathrm{a}}$ & {$[16,35]$} \\
$\gamma p \rightarrow \rho^{0} p$ & $7.0-8.0$ & $2.3-2.9$ & $7.9 \pm 0.3^{\mathrm{b}}$ & {$[14]$} \\
$\gamma p \rightarrow \phi p$ & $4.0-7.5$ & $0.6-2.4$ & $6.86 \pm 0.22$ & {$[36]$} \\
$\gamma p \rightarrow K(892)^{+} \Lambda$ & $4.2-8.1$ & $0.7-2.6$ & $6.65 \pm 0.38$ & {$[41]$} \\
$\gamma p \rightarrow K(892)^{+} \Sigma^{0}$ & $4.3-7.9$ & $0.7-2.4$ & $7.34 \pm 0.45$ & {$[41]$} \\
$\gamma p \rightarrow f_{1}(1285) p$ & $6.0-7.6$ & $1.2-2.0$ & $7.19 \pm 0.96$ & {$[33]$} \\
\hline \hline
\end{tabular}

${ }^{\text {a This result is performed for higher energy } s \text { range. }}$

${ }^{\mathrm{b}}$ This best-fit result taken from Ref. [14].

new CLAS data only [21], performed with a power function $\propto s^{-(n-2)}$, leading to $(n-2)=6.89 \pm 0.26$ (Table I).

The previous CLAS study for $\rho^{0}[14]$ and $\omega$ [16] resulted in $(n-2)=7.9 \pm 0.3$ and $7.2 \pm 0.7$, respectively. Mesons were identifies via the $\rho^{0} \rightarrow \pi^{+} \pi^{-}$and $\omega \rightarrow \pi^{+} \pi^{-} \pi^{0}$ channels. Note that the database for these analyses was limited by $s=6.8-8.4 \mathrm{GeV}^{2}$ and divided into 3-4 energy bins. Then the joint analysis of the CLAS [37] and SLAC [12] cross sections for for the reaction $\gamma p \rightarrow K^{+} \Lambda$ covering the range $s=4.6-12.2 \mathrm{GeV}^{2}$ gave $(n-2)=7.1 \pm 0.1$ [18]. All these CLAS results are consistent with $s^{-7}$.

For our analysis, we included a number of light-meson photoproduction data sets produced by the CLAS Collaboration on the proton and neutron for incident photon energies above $s=3 \mathrm{GeV}^{2}$, carried out during the past 20 years. For one particular case, the $\gamma p \rightarrow \pi^{+} n$ analysis, we included JLab Hall A [15] and SLAC [12] measurements. The results (Fig. 1 and Table I) are consistent with the $(n-2)=7$ (see Fig. 2) scaling expected from the QCR. Oscillations observed at low energies indicate that the QCR requires higher energies and higher $|t|$ and $|u|$ before it can provide a valid description. Obviously, the extended energy range would be more definitive; our results do appear to be consistent with this limit. The JLab12, EIC, and EicC programs are capable of providing the data needed to improve our results.

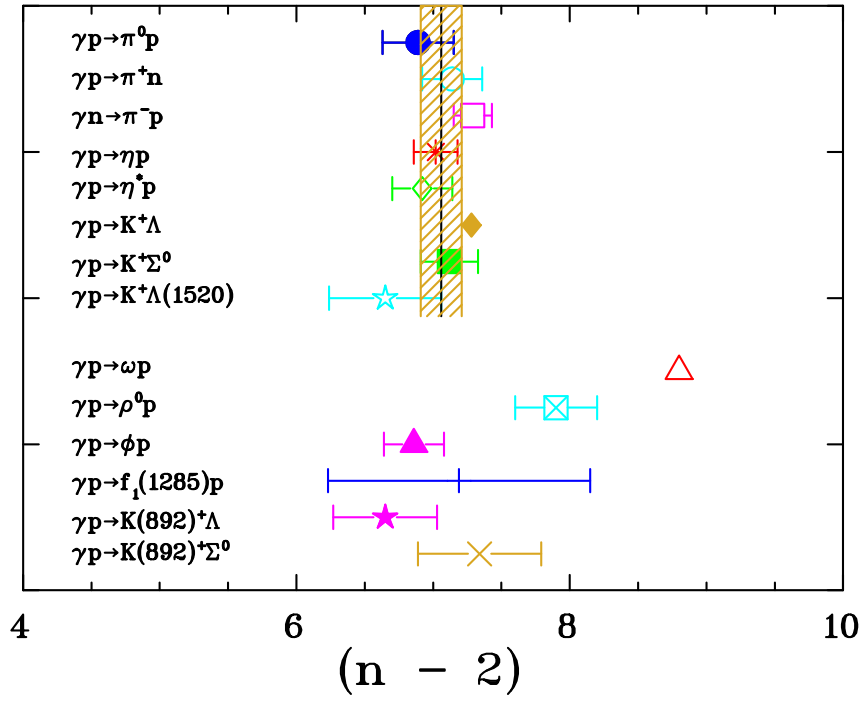

FIG. 2. Power factor $(n-2)$ in Eq. (1) for light-meson photoproduction off the nucleon from the CLAS Collaboration. The black solid vertical line shows the average value for pseudoscalar mesons $\langle(n-2)\rangle=7.06 \pm 0.15$. The yellow band represents its uncertainty. In the case of the $\omega$, the result corresponds to the higher energy range, $s=5-8.1 \mathrm{GeV}^{2}$. The notation for the different reactions is the same as in Fig. 1.

Recently, the analysis of the CLAS $\gamma p \rightarrow \eta \prime p, \gamma p \rightarrow$ $K^{+} \Lambda$, and $\gamma p \rightarrow K^{+} \Sigma^{0}$ [19] data covering a limited energy range of $s=6.2-8.1 \mathrm{GeV}^{2}$ showed that the energy behavior of the $90^{\circ}$ cross section is $d \sigma / d t(s) \propto s^{-7}$. In the cases of $\gamma p \rightarrow \eta p, \gamma p \rightarrow \omega p$, and $\gamma p \rightarrow \phi p$, results are $(n-2)=$ $12.7 \pm 1.2,(n-2)=9.4 \pm 0.1$, and $(n-2)=12.3 \pm 0.6$, respectively. Mesons were identified via $\eta \rightarrow \pi^{+} \pi^{-} \pi^{0}, \omega \rightarrow$ $\pi^{+} \pi^{-} \pi^{0}$, and $\phi \rightarrow K^{+} K^{-}$, respectively. Then the analysis of the $\omega$ photoproduction data [35] for $s=5-8 \mathrm{GeV}^{2}$ results $(n-2)=9.08 \pm 0.11$ [22].

The A2 Collaboration at MAMI reported differential cross sections for $\gamma p \rightarrow \omega p$ close to threshold [42]. The $\omega$ meson was identified via a radiative decay mode $\omega \rightarrow \pi^{0} \gamma$. As Fig. 4 of Ref. [42] shows, there is a disagreement between CLAS and A2 measurements below $s=3 \mathrm{GeV}^{2}$.

The difference between our analysis and analysis by Dey [19] who obtained a larger power $(n-2)$ for the reactions $\gamma p \rightarrow \eta p, \gamma p \rightarrow \omega p$, and $\gamma p \rightarrow \phi p$ is understandable due to different energy ranges of the data included in the fits. Indeed, as one can see in Fig. 1 for these reactions, there is a steeper

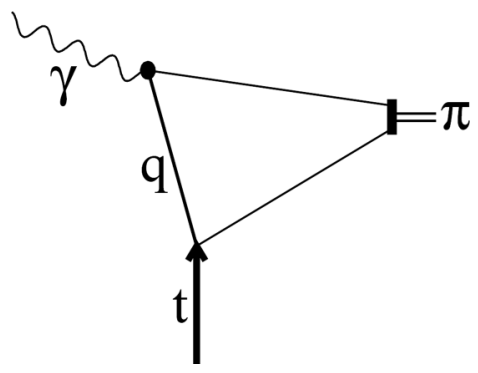

FIG. 3. Simplest diagram for the large $t$ meson photoproduction. 

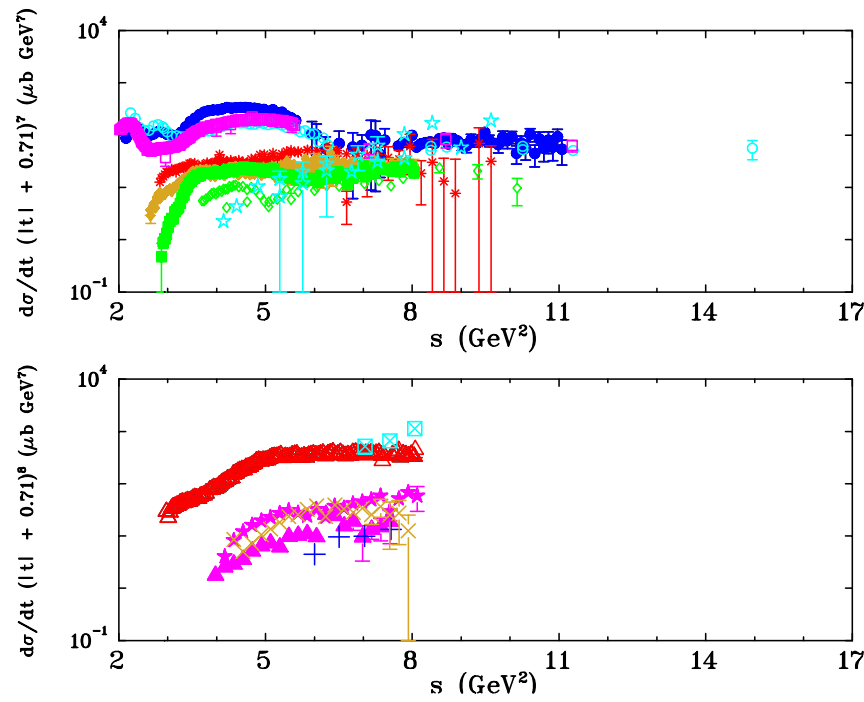

FIG. 4. Differential cross section of the light-meson production off the nucleon at meson production angle $\theta=90^{\circ}$ in c.m. as a function of c.m. energy squared $s$. Upper panel: Pseudoscalarmeson $d \sigma / d t \times(t-0.71)^{7}$. Lower panel: Vector-meson $d \sigma / d t \times$ $(t-0.71)^{8}$. The notation for the different reactions is the same as in Fig. 1 .

energy dependence of the higher $s$ part of the distribution. For the case of the $\phi$ (and partly $\eta$ ) photoproduction, this can be considered as a hint in favor of the noticeable role of the five quark $(u u d s \bar{s})$ component in the proton wave function. Having such a component, the process can be considered as the constituent strange quark interchange between the proton and the $\phi$ meson. However, this explanation is not directly applicable to the $\omega$ photoproduction. Note, however, that, as was mentioned in Ref. [22], due to the vector nature of the $\omega$ meson in order to form the spin part of the corresponding wave function, we have to violate the $s$-channel helicity conservation (SCHC). Therefore, we have to expect an additional suppression of $90^{\circ}$ high energy photoproduction [3]. That is, for the case of the $\rho, \omega$, and $\phi$ mesons the expected power $(n-2)$ should be 1 (for the case of Fig. 3) or 2 (for the case of Fig. 8 of Ref. [22]) units larger). Thus we can say that the observed energy dependence of the $\rho$ cross section and the behavior of $\omega$ at a larger $s$ is consistent with the QCR.

Recall that in the case of $\gamma p \rightarrow \omega p$ and $\gamma p \rightarrow \phi p$, both analyses (our and [19]) used the same experimental data. This indicates the necessity of more experimental data in a wider energy range, especially for the $\rho$ and $\omega$ mesons' photoproduction, to better study the energy dependence and the role of the meson spin and to obtain a more stringent constraint on the fit parameters. It will be interesting also to measure and compare the angular behavior to study the elementary quark-quark hard collision.

In particular, it is important to compare the $\rho$ and $\omega$ vector meson photoproduction in a larger energy interval. First, the $s=7-8 \mathrm{GeV}^{2}$ interval, where the $\rho$ cross section was measured up to now [14], is not sufficient to reliably determine the power $(n-2)$. Note also that, fitting the $\omega$ data at a larger $s=5-8 \mathrm{GeV}^{2}$, we get a larger value of $(n-2)=8.80 \pm 0.06$

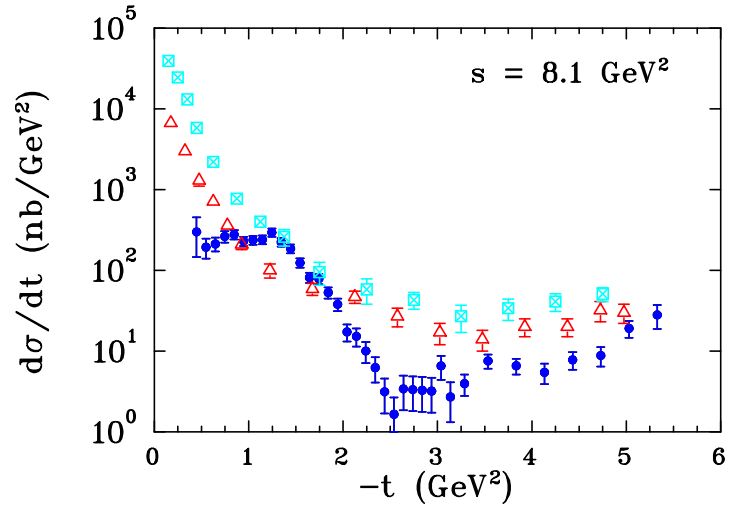

FIG. 5. Differential cross section at $s=8.1 \mathrm{GeV}^{2}$ for the reaction $\gamma p \rightarrow \pi^{0} p$ for $|t|=0.5-5.3 \mathrm{GeV}^{2}\left(90^{\circ}\right.$ corresponds to $|t|=$ $3.2 \mathrm{GeV}^{2}$ ) shown by the blue filled circles [21], for the reaction $\gamma p \rightarrow \omega p$ for $|t|=0.2-5.0 \mathrm{GeV}^{2}\left(90^{\circ}\right.$ corresponds to $|t|=$ $3.0 \mathrm{GeV}^{2}$ ) shown by red open triangles [16], and for the reaction $\gamma p \rightarrow \rho p$ for $|t|=0.2-4.7 \mathrm{GeV}^{2}\left(90^{\circ}\right.$ corresponds to $|t|=$ $3.0 \mathrm{GeV}^{2}$ ) shown by cyan open squares with crosses [14].

which is consistent with results of Refs. [19,22] and the QCR prediction $[(n-2)=9]$ for the process with SCHC violation.

Next the ratio of $\sigma(\rho) / \sigma(\omega)$ cross sections at the moment looks a bit strange. If the photon first produces the $q \bar{q}$ pair then we expect $\sigma(\rho) / \sigma(\omega)=9$, like in diffractive photoproduction. Contrarily, if the photon interacts with one of the proton's valence quarks then the ratio $\sigma(\rho) / \sigma(\omega)=(5 / 3)^{2}$. However, as seen in Fig. 4 at $s=7 \mathrm{GeV}^{2}$, we observe $\sigma(\rho) \simeq \sigma(\omega)$.

An additional interesting fact is that the $\phi, f_{1}(1285)$, and $K(892)^{+}$cross sections are close to each other (Fig. 4).

Since we consider not very large $s$, we have to discuss the possible power corrections to the QCR. Unfortunately, the corresponding power corrections are closely related to the nonperturbative structure of incoming hadrons. Therefore, we evaluate the possible role/scale of the power corrections based on the well known dipole behavior of the proton QED form factor $G(t)=\left(1-t / 0.71 \mathrm{GeV}^{2}\right)^{2}$, which describes all four-momentum dependencies of both electric and magnetic form factors of the proton quite well [43], where the constant $0.71 \mathrm{GeV}^{2}$ determines the scale of the correction in comparison with the asymptotic behavior $G(t)=1 / t^{2}$. It appears natural to introduce a similar "infrared cutoff" at a lower $s$ in our case as well. Thus in Fig. 4 (upper panel), we plot the product $d \sigma / d t(s) \times(t-0.71)^{7}$ for the pseudoscalar mesons. As can be seen, now the $s$ behavior of this product is rather flat down to $s=2-3 \mathrm{GeV}^{2}$. In the lower panel for the vector mesons, we plot the product $d \sigma / d t(s) \times(t-0.71)^{8}$. Here we see the flat $\omega$ cross section dependence for $s>5 \mathrm{GeV}^{2}$. Additionally, Fig. 4 shows that the accuracy (and dispersion) of the data points is better seen here than in Fig. 1 and it demonstrates the possible role of the "infrared cutoff" $(t-0.71)$ in this energy interval.

In Fig. 5, the differential cross section $d \sigma / d t$ is plotted as a function of the $t$ Mandelstam variable at $s=8.1 \mathrm{GeV}^{2}$. The red open triangles are measured data points of the $\gamma p \rightarrow$ $\omega p$ reaction [16] and cyan open squares with crosses of the $\gamma p \rightarrow \rho p$ reaction [14], while the blue solid circles are $\pi^{0}$ 
photoproduction data points [21]. As one can see for the pseudoscalar-meson production at lower values of $t$, the cross section of $\omega$ and $\rho$ photoproduction is an order of magnitude higher than that of $\pi^{0}$ photoproduction; however, at higher values of $t$, the $\omega$ photoproduction cross section is in general still higher but the difference is not as dramatic as at lower values of $t$. It has to be mentioned that data in this figure are for all meson production angles. When only the $90^{\circ}$ production angle data are selected, these three cross sections at higher values of $s$ reach the same level, as do other meson production data, except for the $\phi$ and the $f_{1}(1285)$ photoproduction cross sections, which lie significantly below the other mesons' plateau at higher energies (Fig. 4).

It is interesting to see that the $\phi$ and $f_{1}(1285)$ production cross sections at higher energies and the $90^{\circ}$ production angle are equal to each other within statistical errors, which may indicate a common mechanism of their production. While the $\phi$ has definitely $s \bar{s}$ quark structure, the $K \bar{K} \pi$ branching ratio of $f_{1}(1285)$ is on the order of $10 \%$ which means that on average the $s \bar{s}$ component of the wave function of the $f_{1}(1285)$ is small. However, it seems that, as the presented data may indicate, the $s \bar{s}$ component of the $f_{1}(1285)$ wave function becomes dominant in the hard scattering process, when all three Mandelstam variables $s, t$, and $u$ are large.

\section{SUMMARY AND CONCLUSIONS}

In the present paper, we study the energy dependence of the $90^{\circ}$ light-meson photoproduction off the nucleon. We consider practically all available experimental data obtained by the CLAS Collaboration over more than the last two decades and compare the results with the quark counting rules predictions. We emphasize that in the case of photoproduction the QCR prediction is not affected by the Sudakov form factor. This fact allows a more direct interpretation of the observed results.

Thanks to the pointlike nature of the photon, the $90^{\circ}$ cross section $d \sigma / d t \propto s^{-7}$ for the pseudoscalar-meson production. The average value of $\langle(n-2)\rangle$ for pseudoscalar-meson reactions listed in Table I (top) is $7.06 \pm 0.15$. The explanation of the $s^{-7}$ instead of $s^{-8}$ or $s^{-6}$ is
(1) In terms of Brodsky-Farrar [1]: In photoproduction amplitude, the balance between the quarks momenta was provided by the highly virtual quark with propagator $1 / \hat{q} \propto 1 / \sqrt{s}$ (Fig. 3) instead of the gluon for which the propagator is $\propto 1 / s$.

(2) It terms of Matveev et al. [2]: In photoproduction, the incoming $q \bar{q}$ pair is produced (in the case of a large momentum transferred) very close to the interaction point and not in advance (at a large distance) as in the vector-meson dominance model. That is in the incoming state, we deal with a "pointlike" $q \bar{q}$ pair and only in the final state do we have two quarks separated by large $(\approx 0.5-1 \mathrm{fm})$ distances. The small factor corresponding to the probability of having two quarks very close to each other is needed now for the final state only (and not for the initial state). This leads to the root square of the usual $1 / s^{2}$ factor.

Let us note that the cross section for the light meson photoproduction off the nucleon at $90^{\circ}$ is very small (minimal) and for that reason it may cause a problem for the best-fit analysis using Eq. (2).

Obviously, the JLab6 program is limited by $s \leqslant 11 \mathrm{GeV}^{2}$. Within the JLab12 program, the proposal E12-14-005 for Hall $\mathrm{C}$ approved by JLab PAC can extend the measurement of the $\gamma p \rightarrow \pi^{0} p$ reaction up to $s \leqslant 20 \mathrm{GeV}^{2}$ [44]. Additionally, recent GlueX Collaboration reports allow us to expect data for $\gamma p \rightarrow \eta p[45]$ and $\gamma p \rightarrow \omega p$ [46].

It would be interesting to measure the exclusive large angle photoproduction on a nucleus in order to check that indeed the meson is produced in the pointlike configuration and therefore, thanks to the color transparency effect, it experiences minimal absorption in a nuclear medium [47,48] (see Ref. [49] for a review and references therein).

\section{ACKNOWLEDGMENTS}

We thank Stan Brodsky, Boris Kopeliovich, Shunzo Kumano, Fred Myhrer, and Bernard Pire for useful remarks and discussions. This work was supported in part by the by the US Department of Energy, Office of Science, Office of Nuclear Physics, under Grants No. DE-SC0016583 and No. DE-FG02-96ER40960.
[1] S. J. Brodsky and G. R. Farrar, Scaling Laws at Large Transverse Momentum, Phys. Rev. Lett. 31, 1153 (1973).

[2] V. A. Matveev, R. M. Muradian, and A. N. Tavkhelidze, Automodellism in the large-angle elastic scattering and structure of hadrons, Lett. Nuovo Cimento 7, 719 (1973).

[3] G. P. Lepage and S. J. Brodsky, Exclusive processes in perturbative quantum chromodynamics, Phys. Rev. D 22, 2157 (1980).

[4] D. W. Sivers, Issues in large-angle scattering, Ann. Phys. (NY) 90, 71 (1975).

[5] J. Botts and G. F. Sterman, Hard elastic scattering in QCD: Leading behavior, Nucl. Phys. B 325, 62 (1989).

[6] J. Botts and G. F. Sterman, Sudakov effects in hadron hadron elastic scattering, Phys. Lett. B 224, 201 (1989); Erratum: 227, 501 (1989).

[7] A. Radyushkin, Quark counting rules: Old and new approaches, Int. J. Mod. Phys. A 25, 502 (2010).
[8] K. A. Jenkins, L. E. Price, R. Klem, R. J. Miller, P. Schreiner, M. L. Marshak, E. A. Peterson, and K. Ruddick, Measurement of wide angle elastic scattering of pions and protons off protons, Phys. Rev. D 21, 2445 (1980).

[9] C. Baglin et al. (Annecy (LAPP)-CERN-Bohr Inst-GenoaOslo-London Collaboration), An experimental study of largeangle elastic scattering of charged mesons and antiprotons on protons at 20 and $30 \mathrm{GeV} / \mathrm{c}$ incident momenta, Nucl. Phys. B 216, 1 (1983).

[10] C. White et al., Comparison of 20 exclusive reactions at large $t$, Phys. Rev. D 49, 58 (1994).

[11] T. A. Armstrong et al. (Fermilab E760 Collaboration), Twobody neutral final states produced in antiproton-proton annihilations at $2.911 \lesssim \sqrt{s} \lesssim 3.686 \mathrm{GeV}$, Phys. Rev. D 56, 2509 (1997). 
[12] R. L. Anderson, D. Gustavson, D. Ritson, G. A. Weitsch, H. J. Halpern, R. Prepost, D. H. Tompkins, and D. E. Wiser, Measurements of exclusive photoproduction processes at large values of $t$ and $u$ from 4 to 7.5-GeV, Phys. Rev. D 14, 679 (1976).

[13] D. A. Jenkins and I. I. Strakovsky, Nucleon helicity in pion photoproduction, Phys. Rev. C 52, 3499 (1995).

[14] M. Battaglieri et al. (CLAS Collaboration), Photoproduction of the $\rho^{0}$ Meson on the Proton at Large Momentum Transfer, Phys. Rev. Lett. 87, 172002 (2001).

[15] L. Y. Zhu et al. (Jefferson Lab Hall A Collaboration), CrossSection Measurement of Charged Pion Photoproduction from Hydrogen and Deuterium, Phys. Rev. Lett. 91, 022003 (2003).

[16] M. Battaglieri et al. (CLAS Collaboration), Photoproduction of the Omega Meson on the Proton at Large Momentum Transfer, Phys. Rev. Lett. 90, 022002 (2003).

[17] W. Chen et al. (CLAS Collaboration), A Measurement of the Differential Cross Section for the Reaction $\gamma n \rightarrow \pi^{-} p$ from Deuterium, Phys. Rev. Lett. 103, 012301 (2009).

[18] R. A. Schumacher and M. M. Sargsian, Scaling and resonances in elementary $K^{+} \Lambda$ photoproduction, Phys. Rev. C 83, 025207 (2011).

[19] B. Dey, Scaling behavior in exclusive meson photoproduction from Jefferson Lab at large momentum transfers, Phys. Rev. D 90, 014013 (2014).

[20] K. J. Kong, T. K. Choi, and B. G. Yu, Role of $\omega$-meson exchange in scaling of the $\gamma p \rightarrow \pi^{0} p$ process from a Regge-type model with resonances, Phys. Rev. C 94, 025202 (2016).

[21] M. C. Kunkel et al. (CLAS Collaboration), Exclusive photoproduction of $\pi^{0}$ up to large values of Mandelstam variables $s, t$, and $u$ with CLAS, Phys. Rev. C 98, 015207 (2018).

[22] T. Reed, C. Leon, F. Vera, L. Guo, and B. Raue, The constituent counting rule and omega photoproduction, arXiv:2005.13067.

[23] W. C. Chang, S. Kumano, and T. Sekihara, Constituentcounting rule in photoproduction of hyperon resonances, Phys. Rev. D 93, 034006 (2016).

[24] Yu. L. Dokshitzer, V. A. Khoze, A. H. Mueller, and S. I. Troian, Basics of Perturbative QCD (Edition Frontieres, Gif-sur-Yvette, France, 1991).

[25] J. P. Ralston and B. Pire, Oscillatory Scaling Violation and the Chromodynamics Coulomb phase, Phys. Rev. Lett. 49, 1605 (1982).

[26] B. Pire and J. P. Ralston, Fixed angle elastic scattering and the Chromo-Coulomb phase shift, Phys. Lett. B 117, 233 (1982).

[27] G. R. Farrar, G. F. Sterman, and H. y. Zhang, Absence of Sudakov Factors in Large Angle Photoproduction and Compton Scattering, Phys. Rev. Lett. 62, 2229 (1989).

[28] M. Dugger et al. (CLAS Collaboration), $\pi^{0}$ photoproduction on the proton for photon energies from 0.675 to $2.875 \mathrm{GeV}$, Phys. Rev. C 76, 025211 (2007).

[29] M. Dugger et al. (CLAS Collaboration), $\pi^{+}$photoproduction on the proton for photon energies from 0.725 to $2.875 \mathrm{GeV}$, Phys. Rev. C 79, 065206 (2009).

[30] P. T. Mattione et al. (CLAS Collaboration), Differential cross section measurements for $\gamma n \rightarrow \pi^{-} p$ above the first nucleon resonance region, Phys. Rev. C 96, 035204 (2017).

[31] T. Hu et al. (CLAS Collaboration), Photoproduction of $\eta$ mesons off the proton for $1.2<E_{\gamma}<4.7 \mathrm{GeV}$ using CLAS at Jefferson Laboratory, Phys. Rev. C 102, 065203 (2020).
[32] M. Williams et al. (CLAS Collaboration), Differential cross sections for the reactions $\gamma p \rightarrow p \eta$ and $\gamma p \rightarrow p \eta$, Phys. Rev. C 80, 045213 (2009).

[33] R. Dickson et al. (CLAS Collaboration), Photoproduction of the $f_{1}$ (1285) meson, Phys. Rev. C 93, 065202 (2016).

[34] C. Sowa, Study of excited $\eta$ mesons in photoproduction at CLAS, Ph.D. thesis, Ruhr-University Bochum, 2016 (unpublished).

[35] M. Williams et al. (CLAS Collaboration), Differential cross sections and spin density matrix elements for the reaction $\gamma p \rightarrow$ p $\omega$, Phys. Rev. C 80, 065208 (2009).

[36] B. Dey et al. (CLAS Collaboration), Data analysis techniques, differential cross sections, and spin density matrix elements for the reaction $\gamma p \rightarrow \phi p$, Phys. Rev. C 89, 055208 (2014).

[37] M. E. McCracken et al. (CLAS Collaboration), Differential cross section and recoil polarization measurements for the $\gamma p \rightarrow K^{+} \Lambda$ reaction using CLAS at Jefferson Lab, Phys. Rev. C 81, 025201 (2010).

[38] B. Dey et al. (CLAS Collaboration), Differential cross sections and recoil polarizations for the reaction $\gamma p \rightarrow K^{+} \Sigma^{0}$, Phys. Rev. C 82, 025202 (2010).

[39] K. Moriya et al. (CLAS Collaboration), Differential photoproduction cross sections of the $\Sigma^{0}(1385), \Lambda(1405)$, and $\Lambda(1520)$, Phys. Rev. C 88, 045201 (2013).

[40] U. Shrestha et al. (CLAS Collaboration), Differential cross sections for $\Lambda(1520)$ using photoproduction at CLAS, Phys. Rev. C 103, 025206 (2021).

[41] W. Tang et al. (CLAS Collaboration), Cross sections for the $\gamma p \rightarrow K^{\star+} \Lambda$ and $\gamma p \rightarrow K^{\star+} \Sigma^{0}$ reactions measured at CLAS, Phys. Rev. C 87, 065204 (2013).

[42] I. I. Strakovsky et al. (A2 Collaboration at MAMI), Photoproduction of the $\omega$ meson on the proton near threshold, Phys. Rev. C 91, 045207 (2015).

[43] F. Halzen and A. D. Martin, Quarks and Leptons: An Introductory Course In Modern Particle Physics (Wiley, New York, 1984).

[44] Wide angle exclusive photoproduction of $\pi^{0}$ mesons, spokespersons: D. Dutta, H. Gao, S. Sirca, M. Amaryan, M. Kunkel, and I. Strakovsky (Rcs And Nps Collaborations), JLab Proposal E12-14-005, Newport News, VA, USA, 2014, https:// www.jlab.org/exp_-prog/proposals/14/PR12-14-005.pdf.

[45] M. Kamel (for GlueX Collaboration), Differential $\eta$ photoproduction cross sections off the proton from the GlueX experiment, talk at the 9th Workshop of the APS Topical Group on Hadronic Physics, online, April, 2021, https://indico. jlab.org/event/412/contributions/7792/attachments/6733/9207/ GHP_April2021.pdf.

[46] M. Dalton (for GlueX Collaboration), Probing meson photoproduction mechanisms with observables in exclusive vector-meson photoproduction, talk at the 9th Workshop of the APS Topical Group on Hadronic Physics, online, April, 2021, https://indico.jlab.org/event/412/contributions/7724/ attachments/6748/9203/Dalton_GHP2021_vectormeson.pdf.

[47] G. Bertsch, S. J. Brodsky, A. S. Goldhaber, and J. F. Gunion, Diffractive Excitation in QCD, Phys. Rev. Lett. 47, 297 (1981).

[48] B. Z. Kopeliovich, L. I. Lapidus, and A. B. Zamolodchikov, Dynamics of color in hadron diffraction on nuclei, Pis'ma Zh. Eksp. Teor. Fiz. 33, 612 (1981) [JETP Lett. 33, 595 (1981)].

[49] D. Dutta, K. Hafidi, and M. Strikman, Color Transparency: Past, present and future, Prog. Part. Nucl. Phys. 69, 1 (2013). 